\title{
A!
}

This is an electronic reprint of the original article.

This reprint may differ from the original in pagination and typographic detail.

Monsivais-Velazquez, Daniel; Bhattacharya, Kunal; Barrio, Rafael A.; Maini, Philip K.; Kaski, Kimmo K.

Dynamics of hierarchical weighted networks of van der Pol oscillators

Published in:

CHAOS

DOI:

$10.1063 / 5.0010638$

Published: 01/12/2020

Document Version

Publisher's PDF, also known as Version of record

Published under the following license:

CC BY

Please cite the original version:

Monsivais-Velazquez, D., Bhattacharya, K., Barrio, R. A., Maini, P. K., \& Kaski, K. K. (2020). Dynamics of hierarchical weighted networks of van der Pol oscillators. CHAOS, 30(12), [123146].

https://doi.org/10.1063/5.0010638

This material is protected by copyright and other intellectual property rights, and duplication or sale of all or part of any of the repository collections is not permitted, except that material may be duplicated by you for your research use or educational purposes in electronic or print form. You must obtain permission for any other use. Electronic or print copies may not be offered, whether for sale or otherwise to anyone who is not an authorised user. 


\section{Dynamics of hierarchical weighted networks of van der Pol oscillators}

Cite as: Chaos 30, 123146 (2020); https://doi.org/10.1063/5.0010638

Submitted: 15 April 2020. Accepted: 27 November 2020 . Published Online: 28 December 2020

(D) Daniel Monsivais-Velazquez, (D) Kunal Bhattacharya, (D) Rafael A. Barrio, (D) Philip K. Maini, and (D) Kimmo K. Kaski
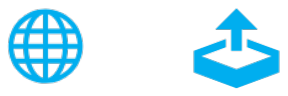

\section{ARTICLES YOU MAY BE INTERESTED IN}

Do reservoir computers work best at the edge of chaos?

Chaos: An Interdisciplinary Journal of Nonlinear Science 30, 121109 (2020); https:// doi.org/10.1063/5.0038163

Theory of quasi-simple dispersive shock waves and number of solitons evolved from a nonlinear pulse

Chaos: An Interdisciplinary Journal of Nonlinear Science 30, 123148 (2020); https:// doi.org/10.1063/5.0028587

On star-convex volumes in 2-D hydrodynamical flows and their relevance for coherent transport

Chaos: An Interdisciplinary Journal of Nonlinear Science 30, 123147 (2020); https:// doi.org/10.1063/5.0028100

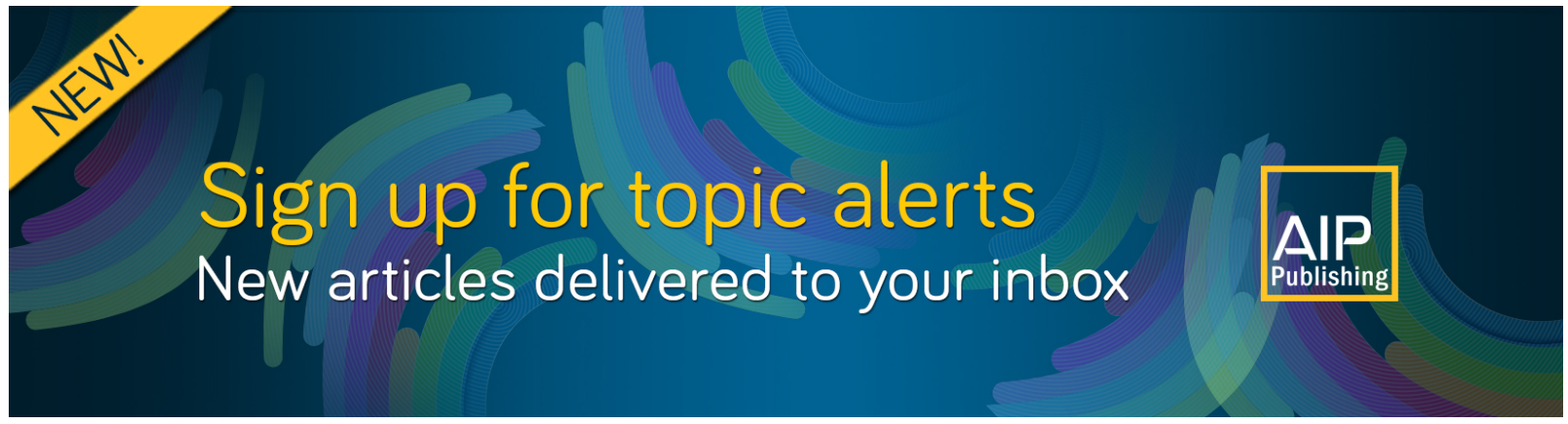




\title{
Dynamics of hierarchical weighted networks of van der Pol oscillators
}

\author{
Cite as: Chaos 30, 123146 (2020); doi: 10.1063/5.0010638 \\ Submitted: 15 April 2020 . Accepted: 27 November 2020. \\ Published Online: 28 December 2020

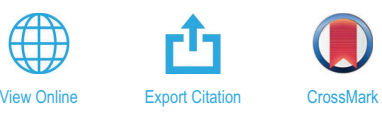

Daniel Monsivais-Velazquez, ' (D) Kunal Bhattacharya, ${ }^{2}$ (D) Rafael A. Barrio, ${ }^{3}$ (D) Philip K. Maini, ${ }^{4}$ (D) and Kimmo K. Kaski ${ }^{1,5, a)}$ (D)

\begin{abstract}
AFFILIATIONS
${ }^{1}$ Department of Computer Science, Aalto University School of Science, 00076 Helsinki, Finland

${ }^{2}$ Department of Industrial Engineering and Management, Aalto University School of Science, 00076 Helsinki, Finland

${ }^{3}$ Instituto de Física, Universidad Nacional Autónoma de México, Ap. postal 01000, CDMX, Mexico

${ }^{4}$ Wolfson Centre for Mathematical Biology, Mathematical Institute, Oxford University, Oxford OX2 6GG, United Kingdom

${ }^{5}$ The Alan Turing Institute, 96 Euston Rd., Kings Cross, London NW1 2DB, United Kingdom
\end{abstract}

${ }^{a}$ Author to whom correspondence should be addressed: kimmo.kaski@aalto.fi

\begin{abstract}
We investigate the dynamics of regular fractal-like networks of hierarchically coupled van der Pol oscillators. The hierarchy is imposed in terms of the coupling strengths or link weights. We study the low frequency modes, as well as frequency and phase synchronization, in the network by a process of repeated coarse-graining of oscillator units. At any given stage of this process, we sum over the signals from the oscillator units of a clique to obtain a new oscillating unit. The frequencies and the phases for the coarse-grained oscillators are found to progressively synchronize with the number of coarse-graining steps. Furthermore, the characteristic frequency is found to decrease and finally stabilize to a value that can be tuned via the parameters of the system. We compare our numerical results with those of an approximate analytic solution and find good qualitative agreement. Our study on this idealized model shows how oscillations with a precise frequency can be obtained in systems with heterogeneous couplings. It also demonstrates the effect of imposing a hierarchy in terms of link weights instead of one that is solely topological, where the connectivity between oscillators would be the determining factor, as is usually the case.
\end{abstract}

Published under license by AIP Publishing. https://doi.org/10.1063/5.0010638

Many oscillatory signaling processes in nature are multi-scale, where the characteristic macro-scale frequency of the signal is an emergent property of the coupling of oscillators of different frequencies acting on the micro-scale. Here, we show, using a combination of numerical simulations and analytical investigations, how oscillations of a precise frequency can be obtained from a fractal network of oscillators when the coupling is heterogeneous. Our results on various hierarchically coupled networks shed light on how the frequencies synchronize and the phases become locked at various scales. Our model also shows how the concept of hierarchy can be realized when studying synchronization in weighted networks.

\section{INTRODUCTION}

Oscillations are commonly observed in various physical, chemical, and biological systems. ${ }^{1}$ Often, these oscillations appear as macro-scale synchronization phenomena. These emerge from the interactions between a large number of oscillatory elements in a system, acting at a lower, micro-scale level..$^{2-4}$ The structure of these systems can be visualized as a network of nodes or oscillators connected to other oscillators with interaction links in a certain topology. ${ }^{5}$ Often, the oscillatory elements are considered to be of Kuramoto, van der Pol, or some other type. The links between each pair of oscillators are either homogeneous or heterogeneous with a certain weight describing the interaction strength between oscillators, and the topology of the network is either random, regular, hierarchical, or of other complex type. ${ }^{5-7}$ The effects of these features on macro-scale synchronization of a network of oscillators are interesting, yet far from being fully understood.

In a pioneering study by Pavlidis, ${ }^{8}$ it was shown that in an arbitrary network of $N$ linearly coupled harmonic oscillators, the fundamental frequency of the system decreases as $1 / \sqrt{N}$. This result is also found to be exact in the case of non-linear dissipative oscillators when the dissipation coefficient is a function of the coordinate only and the coupling is large enough to induce entrainment. However, this feature of a system is not desirable in the case of 
biological or physiological processes producing, for example, the circadian rhythm of a living organism. ${ }^{9,10}$ Therefore, to describe the behavior of these kinds of systems, which show a single fundamental frequency, one needs to go beyond linear network models and introduce more complex interactions. This we believe is important not only for understanding the outcomes of biological and physiological processes, but also for social processes in large scale societal networks, which show regular and synchronized circadian activity patterns. ${ }^{11-16}$ This issue was addressed in the context of celllevel circadian rhythms in an earlier paper by some of us. ${ }^{17}$ There, it was demonstrated that in a model of heterogeneously coupled oscillators with a hierarchy of coupling strengths, its fundamental frequency can be tuned to any desired value lower than the intrinsic single oscillator frequency. In the present study, we generalize the approach of that work to investigate the dynamics of regular and fractal-like heterogeneously coupled oscillator networks with respect to the distribution of coupling strengths.

Models incorporating coupling heterogeneity have been shown to influence the synchronizability of oscillator networks in various ways. For example, the presence of disorder or heterogeneity was observed to replace the synchronized state with periodic firings in pulse-coupled oscillators, ${ }^{18}$ while synchronizability was enhanced in networks with heterogeneous connectivity. ${ }^{19,20}$ Moreover, associating different coupling strengths to different oscillators in the Kuramoto model resulted in the emergence of clusters of synchronized oscillators near the transition. ${ }^{21}$ Recently, in the case of Belousov-Zhavotinsky chemical oscillators, it was found that the parameters that control synchronization are different in the weak and strong coupling regimes. ${ }^{22}$

In this paper, we demonstrate that a model consisting of van der Pol oscillators ${ }^{23-25}$ with heterogeneous couplings, defined in a family of regular hierarchical networks, not only stabilizes the frequency, but also results in phase locking and synchronization, which are properties that are relevant to the modeling of many biological and social processes. ${ }^{26-29}$ The main aim of this work is to show a way by which the characteristic frequency of a system of coupled oscillators could be tuned using the distribution of coupling strengths such that this frequency does not depend on the number of oscillators when their number is large. Beginning with networks having Sierpinski graph-topologies, ${ }^{30}$ we define a hierarchy of coupling strengths (weights) on the links using a recursive relation. To the best of our knowledge, our work is the first that uses this approach. Previous studies on oscillators in hierarchical networks have introduced the hierarchy via topological features, reflected in the adjacency matrix. . $24,31-33^{-1}$

This paper is organized as follows. After the Introduction (Sec. I), we describe our hierarchical network model with different topological structures (Sec. II). Then, we present the numerical solutions for these hierarchical network topologies (Sec. III) and develop a mean-field-like approximate analytical solution (Sec. IV). Finally, in Sec. V, we present our concluding remarks.

\section{THE MODEL}

Our model is based on a previous one formulated by some of the authors of this study, ${ }^{17}$ in which a hierarchically connected group of $N$ oscillators is shown to stabilize the collective frequency of oscillations, while avoiding the universal feature that the frequency decays like $1 / \sqrt{N}$. This is a peculiar phenomenon since a set of linear (or non-linear) oscillators with constant interactions between the nodes of an arbitrary network exhibits a fundamental frequency that follows the dependence $\omega(N)=\omega(0) \sqrt{1-r(N-1)}$, where $r$ is the strength of the elastic interactions and $\omega(0)$ is the fundamental frequency of a single oscillator. Note that if $r \geq(N-1)^{-1}$, then the system becomes unstable.

In our model, we assume the oscillator nodes to be of a nonlinear van der Pol type, ${ }^{34}$ described by the following equation: ${ }^{35}$

$$
\ddot{x}-\mu\left(1-x^{2}\right) \dot{x}+\omega_{0}^{2} x=0,
$$

where $\mu>0$ is the bifurcation parameter, $\omega_{0}$ is a coefficient in units of frequency, and the dot denotes the time derivative. For a system of $N$ identical oscillators coupled with elastic connections $r_{i j}$, the dynamics can be expressed by the following set of equations:

$$
\begin{gathered}
\dot{x}_{i}=\omega_{0} y_{i}, \\
\dot{y}_{i}=-\omega_{0}\left(x_{i}-\sum_{j \in n(i)} r_{i j} x_{j}\right)+\mu\left(1-x_{i}^{2}\right) y_{i},
\end{gathered}
$$

where $n(i)$ is the set of neighbors of an oscillator $i$ placed at a node of a regular graph with fractal-like topology of $L$ levels and $K$ is the coordination number (see the top panel in Fig. 1 for $L=3$ and $K=3$ ). Also, we assume that the couplings are symmetric; i.e., $r_{j i}=r_{i j}$. The fractal-like layout of the network is used to determine the strength of the interactions $r_{i j}$ between neighboring oscillators, assigning them variable weights depending on their relative locations. In essence, the network consists of many units, each one with $K$ nodes that are connected in an all-to-all fashion ( $K$-cliques). These units are, in turn, connected with other units following the same procedure, forming $K$-cliques of units. This recursive process is repeated $L$ times, i.e., the number of levels, to generate a fractal-like connected network, denoted by $\mathbb{S}(L, K)$ and being of size $N=K^{L}$. Here, we follow the nomenclature of the well known finite Sierpinski graphs. ${ }^{30}$ In Fig. 2, we show five topologically different $\mathbb{S}(L, K)$ graphs for coordination numbers, $K=2,3,4,5$, and 6 , each with $L=5$ hierarchical levels or interaction strengths between neighboring oscillators.

The definition of the hierarchical link strength $r_{i j}$ follows a recursive procedure such that the strength of interaction depends on the level $v=L, L-1, \ldots, 1$, at which the link is located and is given by the following relation:

$$
r_{i j}(v)=a q^{L-v}, \quad 0 \leq q \leq 1,
$$

where $a$ is the strength at the deepest level $v=L$ (while $v=1$ represents the shallowest level) and $q$ is a decay parameter of the strength, which does not depend on $v$. Hence, initially, all the links connecting nodes inside a single unit ( $K$-clique) have strength $r_{i j}(v=L)=a$. These links belong to the deepest level $v=L$ of the network and thus are the strongest. The next set of links connecting different cliques, i.e., forming $K$-cliques of $K$-cliques have diminished strength, $r_{i j}(v=L-1)=a q=q r_{i j}(L)$, and constitute the second deepest level $v=L-1$ of the hierarchical network. This process continues until the links connecting the largest possible 

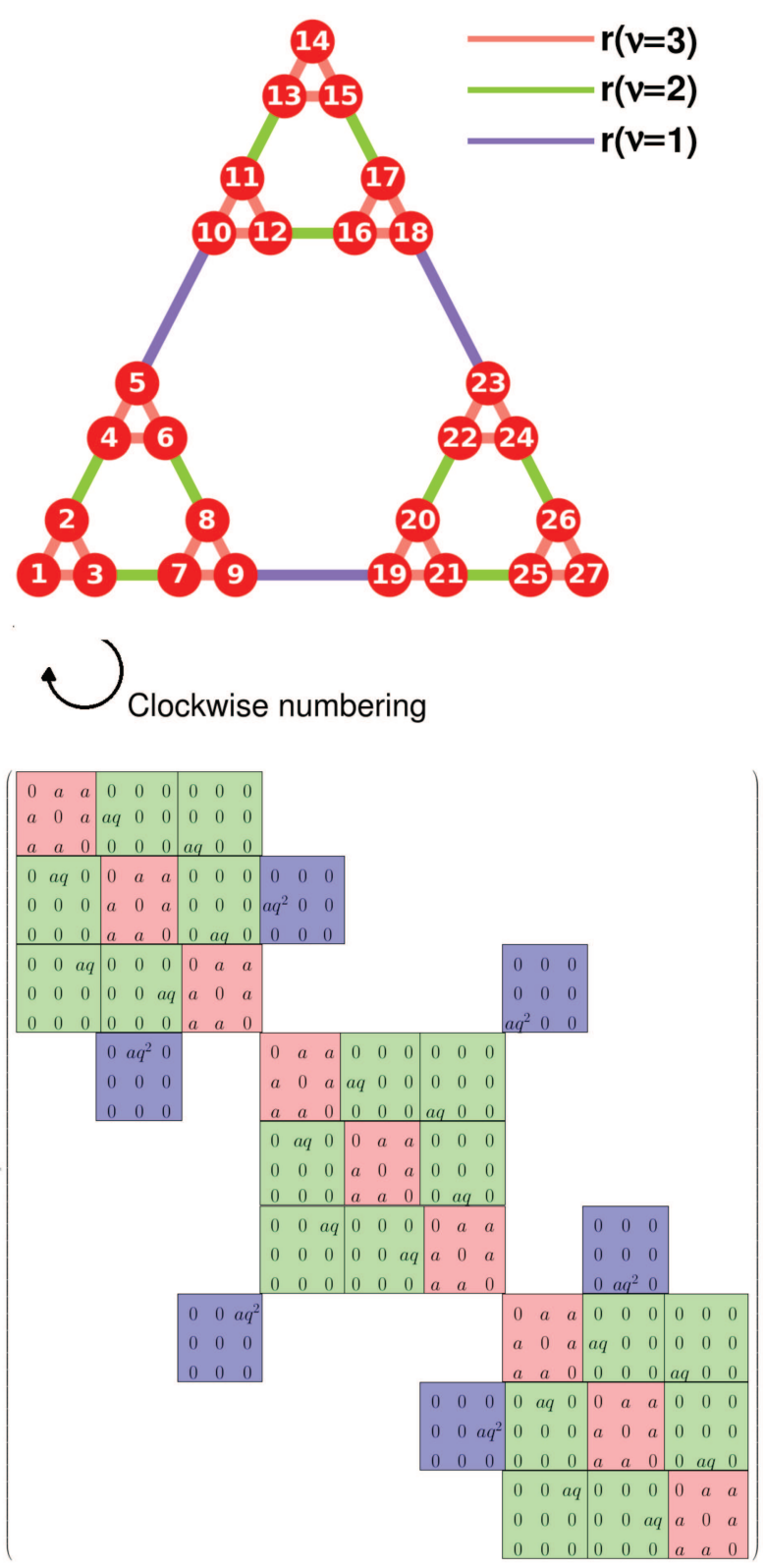

FIG. 1. (Top) Recursively generated fractal-like network with $L=3$ levels and coordination number $K=3$. The network $[S(3,3)]$ has a triangular topology and contains triads of fundamental oscillators as the basic units $K=3$-cliques that are coupled up to the level $L=3$. Links joining oscillators inside each basic unit or clique are colored in pink, and links joining these basic triangular units are colored in green, thus forming yet larger triangular units of basic triangular units, while links joining these larger units of basic units are colored in purple. (Bottom) Adjacency matrix representing the network $[\mathbb{S}(3,3)]$ shown in the top panel. For the number of levels $L=3$, the network has $3^{L}=27$ oscillators. Colored blocks encircle the links between each basic triplet of oscillators and the other elements in the network. The color code is the same as above, with pink, green, and purple representing links within each unit, between units, and between units of units, respectively.

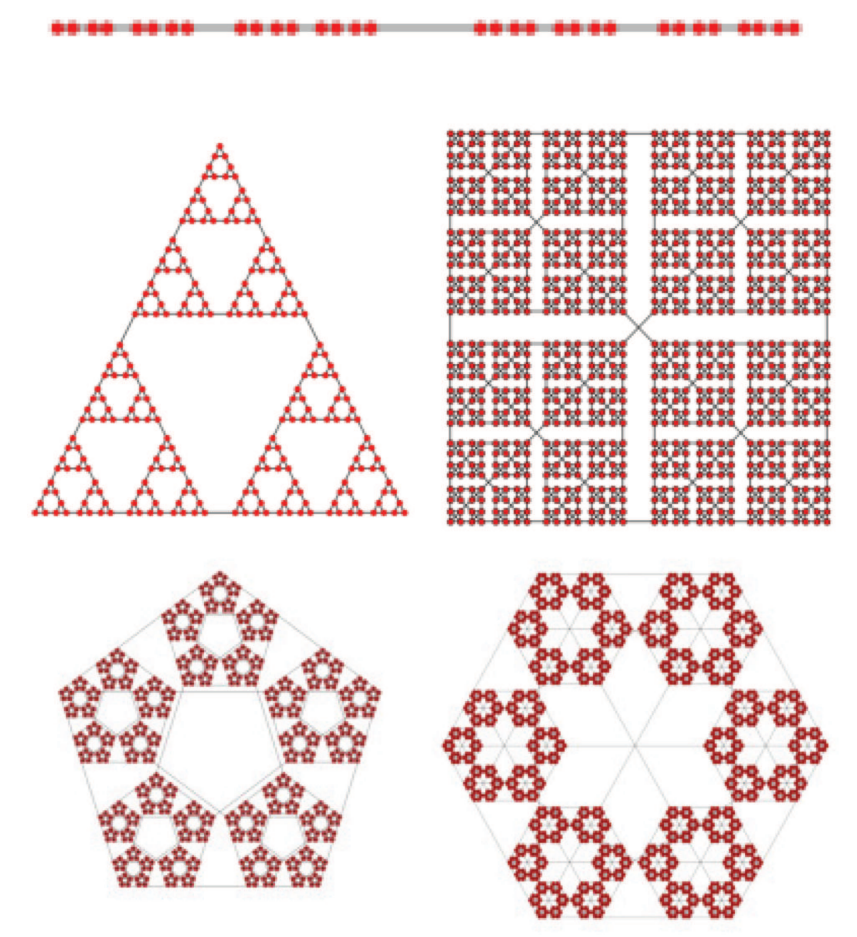

FIG. 2. Examples of five different topologies for Sierpinski graphs $\mathbb{S}(L, K)$ for $L=5$ levels and $K=2,3,4,5$, and 6 : (top) $K=2$ and $2^{L}=32$ nodes; (middle-left) $K=3$ and $3^{L}=243$ nodes; (middle-right) $K=4$ and $4^{L}=1024$ nodes; (bottom-left) $K=5$ and $5^{L}=3125$ nodes; and (bottom-right) $K=6$ and $6^{L}=7776$ nodes

units (the cliques of the cliques of ... of the cliques), constituting the most shallow level $v=1$, which have the weakest interaction strength, i.e., $r_{i j}(v=1)=a q^{L-1}$. In Fig. 1, the connection strengths of a hierarchical network with $K=3$ (i.e., triangles as basic units) are shown, where the strength of connections located at different depth levels $v=3,2,1$ is colored in pink, green, and violet, respectively.

To illustrate the structural properties of our model system, let us consider the example of Fig. 1 for the network $\mathbb{S}(L=3, K=3)$. Here, the three nodes at the three corners of the biggest triangle in Fig. 1 (top) each have one link fewer than the rest of the nodes in the network; i.e., their coordination number is two. Similarly, for the general topology of the finite network, $\mathbb{S}(L, K)$, having $L$ hierarchical levels and coordination number $K$, there are $K$ corner points or nodes with coordination number $(K-1)$ and a total of $\left(K^{L}-K\right)$ nodes with coordination number $K$. Thus, the role of these $K$ corner nodes can be assumed small for large $L$, and they can be used to introduce external forces or perturbations into the system. If we number the oscillators in a clockwise manner within each triangle, then we obtain the weighted adjacency matrix shown in Fig. 1 (bottom).

It is obvious that there is no closed-form analytic solution to the dynamics of the model presented in Eqs. (2a) and (2b) due to its non-linearity and structural complexity. Thus, to describe the 
evolution of the system in time, these equations of motion need to be integrated numerically. To do this, we have chosen the fourth order Runge-Kutta method (RK4), as it has been found to be well suited for integrating systems of coupled oscillators.

\section{NUMERICAL SOLUTION}

We are interested in finding the fundamental frequency of the oscillating units in different regions of the network since these modes are the ones corresponding to the long wavelength excitations and are the modes that persist longer in the network. In order to describe such a process, we follow a coarse-grained analysis.

The process starts in a base network $\mathbb{S}(L, K)$ with $L$ levels and link weights as described before. In this base network, the elements forming the basic $K$-cliques (connected by the strongest connections) are considered to oscillate collectively as a single unit, with frequency $\omega(v=L-1)$, which is, in general, different from the intrinsic frequency of the individual oscillators. Each new unit has an associated output $s^{(\nu)} \equiv s^{(L-1)}$ defined as the sum of the amplitudes of the internal oscillators in the clique forming the unit. The set of oscillating units can be visualized as a network, with topology similar to that of the base network but having one level less and considered a Sierpinski graph $\mathbb{S}(L-1, K)$ coarse-grained from the base network. Following this recursive coarse-graining analysis procedure, in the next step, a new graph is constructed from the previous $\mathbb{S}(L-1, K)$ graph by considering each one of its cliques of nodes as a new single unit with output $s^{(L-2)}$, and then this set of new oscillating units is visualized as a network of Sierpinski graph topology $\mathbb{S}(L-2, K)$ with one level less than the former (i.e., two levels less than the original). This procedure is repeated until the last step of the coarse-graining process is reached; i.e., a $\mathbb{S}(0, K)$ graph (a single node) is generated by clumping together the oscillating units of the previous $\mathbb{S}(1, K)$ graph (a single $K$-clique).

In the method described above, for a given step of the coarsening process, each of its units is generated from a clique of units in the preceding network by clumping them together. In general, for a coarse-grained network $\mathbb{S}(v-1, K)$ with $v-1$ levels, the output $s_{I}{ }^{(\nu-1)}$ of an oscillating unit $I$ is given by the sum of the outputs $s_{i}{ }^{(v)}$ of the oscillating units belonging to the parent network (with $v$ levels) from which this network was coarse-grained; thus,

$$
s_{I}^{(\nu-1)}=\sum_{i \in \text { a clique in } \mathbb{S}(\nu, K)} s_{i}^{\left({ }^{\nu}\right)},
$$

where the sum is taken over the $K$ units in the corresponding clique of the parent network. Observe that the coarse-grained level and the hierarchy level $v$ are the same and that a scaling factor is needed to keep the amplitude value within bounds. An example of this recursive procedure is shown in Fig. 3 , where an initial network $\mathbb{S}(3,3)$ is coarse-grained into a single unit $[\mathbb{S}(0,3)]$ in three coarse-graining steps. In this figure, the process of grouping together outputs from one network into a new output unit in the child network is illustrated.

We study initially the temporal evolution of the system $\mathbb{S}(10,3)$, solving numerically Eqs. (2a) and (2b). The scale of the fundamental frequency of every oscillator in the system was fixed by $\omega_{0}$, the time step of integration with RK4 was set to $d t=0.005 \omega_{0}^{-1}$, and the total time of each simulation was $t_{f}=25$ periods $(5000 d t)$. In the top-left

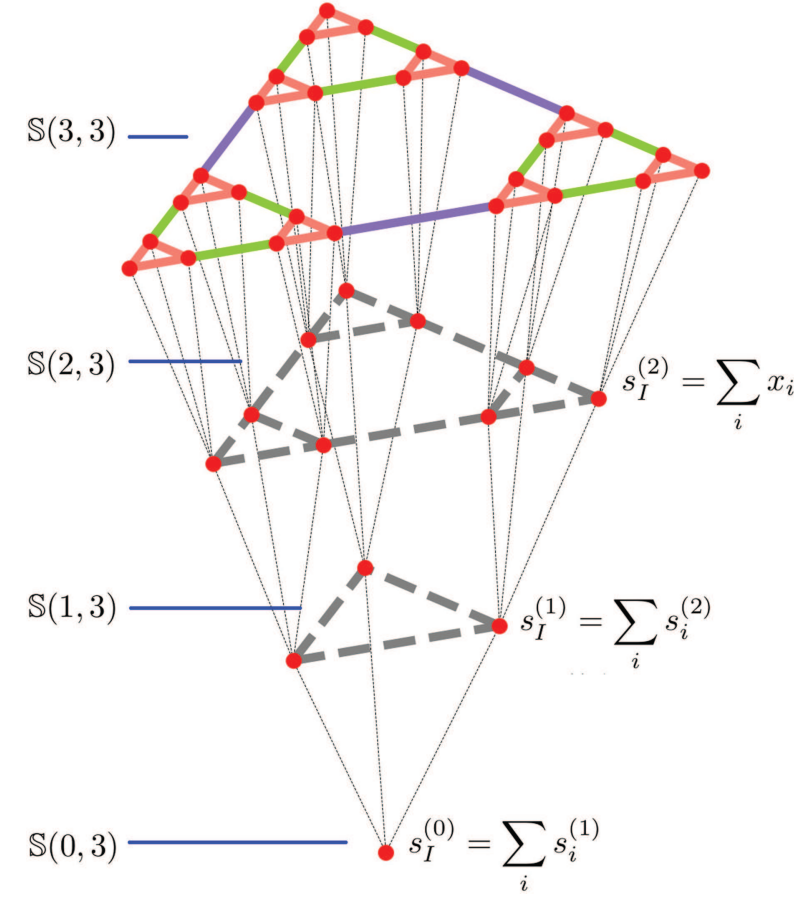

FIG. 3. Illustration of the recursive coarse-graining analysis procedure. Starting from the base network of the Sierpinski graph topology $\mathbb{S}(3,3)$, nodes from each triangle or 3-cliques are grouped together into single nodes. The output $s$, of each new node is the sum of the outputs of the 3-clique oscillators forming the group $\left(s_{l}{ }^{(2)}=\sum_{i} x_{i}\right)$. The resulting nodes are connected following a Sierpinski graph $\mathbb{S}(2,3)$ layout, forming a coarse-grained system one level lower than the previous one. In the next step, nodes belonging to 3-cliques are grouped by adding their outputs, forming a coarse-grained network $\mathbb{S}(1,3)$, with the output of each $/$ node given by $s_{l}{ }^{(1)}=\sum_{i} s_{i}{ }^{(2)}$. Following the same procedure, in the final step, the three nodes are collapsed into a network of one node only, $\mathbb{S}(0,3)$, with output $\left(s_{l}{ }^{(0)}=\sum_{i} s_{i}^{(1)}\right)$. The color code is the same as in Fig. 1, with pink, green, and purple representing links within each unit, between units, and between units of units, respectively.

panel of Fig. 4, we show the temporal evolution (in units of $1 / \omega_{0}$ ) of three oscillators located in one of the corner cliques (with a node with two links and the other two nodes with three links) of the network $\mathbb{S}(10,3)$. We have tuned the parameters of our model system to be $a=0.4, q=0.7$, and $\mu=1$, which stabilizes the final frequency to one half of the fundamental frequency. This configuration resembles a system with two distinguishable rhythms.

Once we had numerically solved the dynamical equations, we applied the coarse-graining process to the system and observed the temporal evolution of the outputs over the recursive process. In the eight panels of Fig. 4, we show the results for the three nodes of the coarse-grained corner clique in the $\mathbb{S}(9,3), \mathbb{S}(8,3), \mathbb{S}(7,3), \mathbb{S}(6,3)$, $\mathbb{S}(4,3), \mathbb{S}(2,3)$, and $\mathbb{S}(0,3)$ networks, respectively. It can be seen that the oscillators reach a state of synchronization in about ten periods (one period is measured in units of $1 / \omega_{0}$ ) and that the coarsegrained network nodes at different levels of hierarchy oscillate with a frequency of about $\omega_{0} / 2$. 

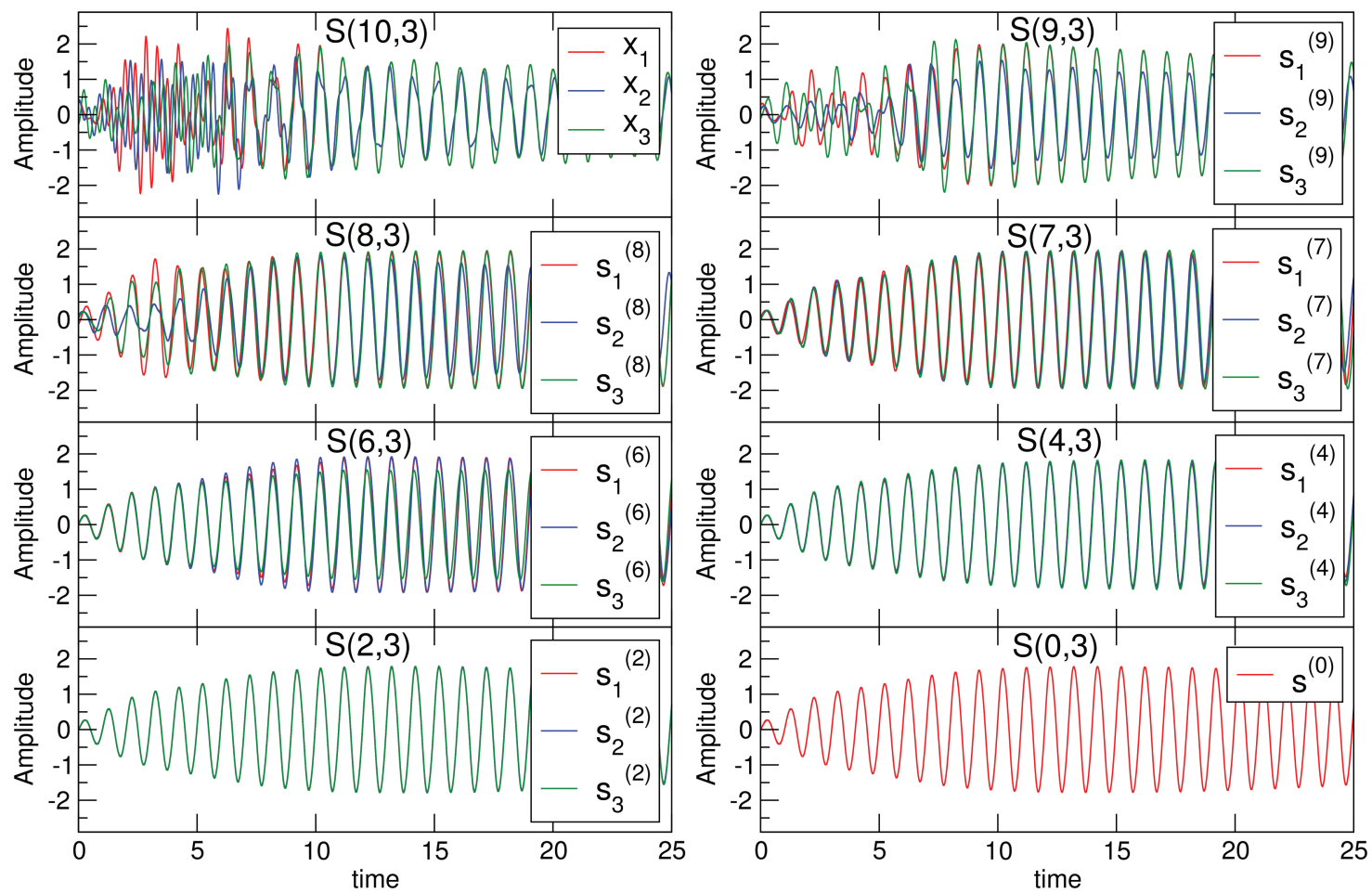

FIG. 4. Time series of the measured signals at specific locations on the networks of different levels obtained during the coarse-graining process. The amplitude of each signal shown was scaled to the interval $[-2,2]$. In the top-left panel, the amplitude $x$ of three different oscillators in a base network $\mathbb{S}(10,3)$ is shown. The signal of three different outputs in the coarse-grained networks $\mathbb{S}(9,3), \mathbb{S}(8,3), \mathbb{S}(7,3), \mathbb{S}(6,3), \mathbb{S}(4,3), \mathbb{S}(2,3)$, and $\mathbb{S}(0,3)$, derived from the base network, are shown in a sequential order. For all these networks, the three nodes chosen for visualization belonged to the first small triangle (from the left) located at one of the three corners of the corresponding network.

The expected dynamics of the coupled system described in Sec. II is that the fractal nature of the network connecting the oscillators induces the system to shift toward a frequency-synchronized state, with its frequency of oscillation tending toward an asymptotic value. This value depends on the baseline frequency coefficient $\omega_{0}$ of each oscillator in the network and on the bifurcation parameter $\mu$ of the van der Pol oscillator, as well as on the network parameters, $a, q, K$, and $L$, i.e., the base level interaction strength, its decay factor, coordination number, and number of levels, respectively, but the asymptotic value of the frequency for sufficiently large networks does not depend on the number of nodes $N$. From Fig. 4, it can be seen that the chosen oscillators of the base network approaches a synchronized state after a transient period. Here, the frequency of oscillations settles to approximately $0.55 \omega_{0}$, which is about one half of the fundamental frequency of the van der Pol oscillator $[\omega(0)]$. Similarly, applying the coarse-graining procedure $L$ times, we end up with the final single node "network" that oscillates with an asymptotic frequency of about one half of the fundamental frequency.

To explore the frequency-synchronization process along the coarse-graining procedure, we have numerically integrated the dynamical equations describing the following five systems: $\mathbb{S}(12,2)$, $\mathbb{S}(10,3), \mathbb{S}(8,4), \mathbb{S}(7,5)$, and $\mathbb{S}(6,6)$ with $4096,59049,65536$, 16384 , and 46656 nodes, respectively. For all these cases, we have set the bifurcation parameter $v=1$ and the RK4 integration time step $d t=0.005 \omega_{0}^{-1}$. The total time $t_{f}$ of the simulations was set to 100 periods $(20000 d t)$, and the initial transient time was set to $t_{0}=25$ periods, such that any quantity averaged over time was calculated for the time interval of $t_{f}-t_{0}=75$ periods. In order to make the system reach an asymptotic frequency of about one half of the fundamental frequency, we need to tune the base level interaction strength $a$ and its decay parameter $q$ for each topology of the hierarchical network. Thus, for $K=2,3,4,5$, and 6 , we have set the strength $a$ to $0.55,0.4,0.3,0.2$, and 0.14 and $q$ to $0.8,0.7,0.7,0.8$, and 0.7 , respectively. All the calculations were done with random initial conditions for $x_{i}(0)=0.5 \zeta_{1}$ and $y_{i}(0)=0.1+0.01 \zeta_{2}$, with $\zeta_{1}$ and $\zeta_{2}$ drawn from a flat distribution in the interval $(-1,1)$. For each network topology, we calculate the mean frequency of oscillation $\bar{\omega}$ at each coarse-graining level $v$, as the average of the frequencies over the set of oscillators/units in the network. The results are shown in Fig. 5, and it can be seen that in all cases, after the coarsening process is applied, the (asymptotic) mean frequency is about one half of the initial frequency. For the hierarchical network topology with $K \leq 3$, we observe that the mean frequency keeps reducing to level 4 , after which it is stabilized to $1 / 2$ of the original frequency for all the remaining hierarchy levels. For other network topologies $(K>3)$, the frequency reaches its asymptotic value faster, after the second level of the coarsening process. 

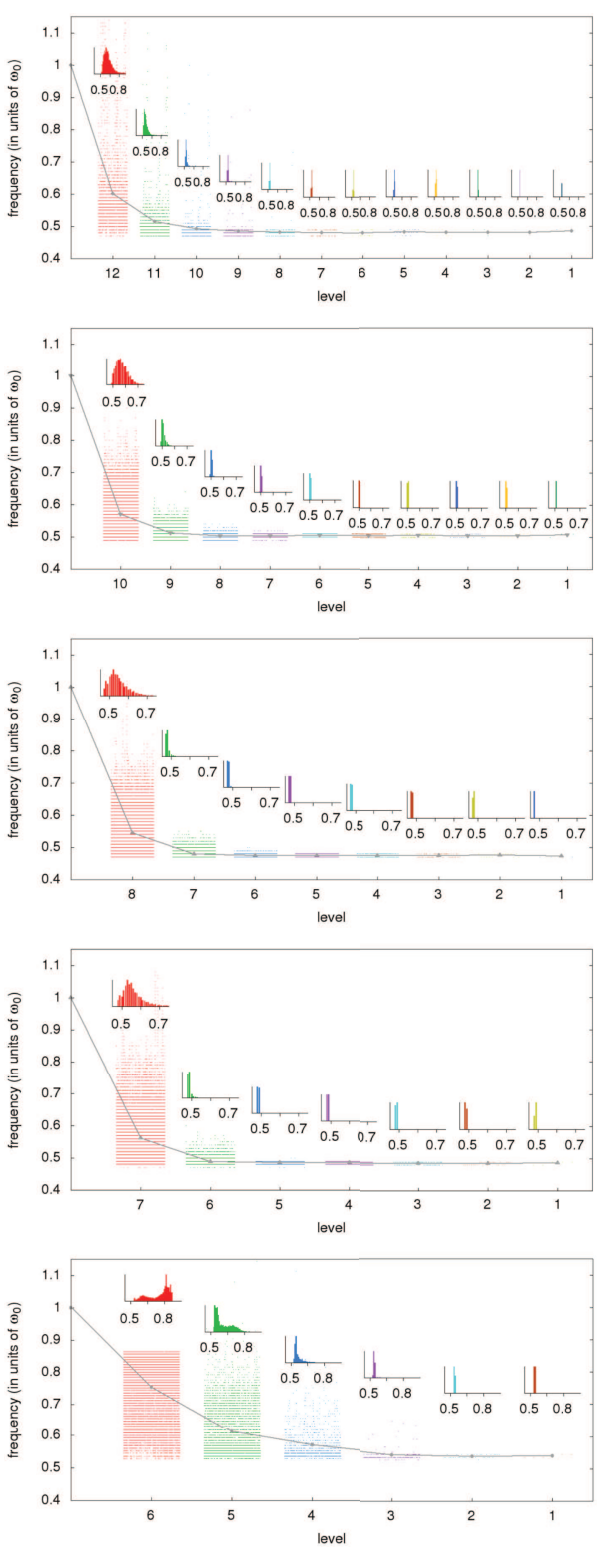

FIG. 5. The mean of the observed frequency $\bar{\omega}$ (in units of $\omega_{0}$ ) as a function of the level $(v)$ in the coarse-grained network (gray line). The distribution of measured frequencies of the outputs for each level is shown in the small histogram above the corresponding mean value, with the horizontal axis representing frequency values (in units of $\omega_{0}$ ). In addition, for each level, the frequency of each output is plotted (dots around the mean value), arranged horizontally according to a clockwise numbering scheme similar to that shown in Fig. 1. The network parameters are (top) $K=2, L=12,4096$ nodes, $a=0.55$, and $q=0.8$; (middle-top) $K=3, L=10,59049$ nodes, $a=0.4$, and $q=0.7$; (middle) $K=4$, $L=8,65536$ nodes, $a=0.3$, and $q=0.7$; (middle-bottom) $K=5, L=7$, 16384 nodes, $a=0.2$, and $q=0.8$; and (bottom) $K=6, L=6,46656$ nodes, $a=0.14$, and $q=0.7$. The asymptotic frequency, for the last step of the coarsegraining process, i.e., the coarse-grained network with just one level, is nearly half of $\omega_{0}$ for all the topologies evaluated. The total time of the simulations was set to 100 periods, with an initial transient time of 25 periods.
To obtain a descriptor of the characteristic frequency of an oscillator belonging to a coarse-grained network with a certain number of levels, we calculate the mean frequency $\bar{\omega}(\nu)$ as the average of the individual frequencies $\omega_{i}^{(v)}$ over the set of all oscillators of the network with $v$ levels, given by

$$
\bar{\omega}(v)=\frac{1}{K^{v}} \sum_{i \in \mathbb{S}(v, K)} \omega_{i}^{(v)},
$$

with $K$ being the coordination number of the network. The frequency $\omega_{i}^{(v)}=2 \pi / T_{i}$ of output $i$ was measured by calculating the average time period $T_{i}$ between pairs of consecutive peaks, and only the second half of the time series was used when calculating the average to exclude the initial transients. We find that oscillators located in different regions of the network have different frequencies such that they are distributed around the mean, with the observed shape and width of the frequency distribution depending on the hierarchy level parameter $v$. At the base level network, $v=L$, the frequencies are distributed within the interval $\left[0.5 \omega_{0}, 0.9 \omega_{0}\right]$ for all the network topologies, as depicted by the leftmost frequency bands (in red) in all the panels of Fig. 5. However, as the system is coarsegrained, the width of the distribution is continuously reduced, and after some steps of the coarse-graining process, the distribution has collapsed into a single value, as seen in all the panels by the frequency bands becoming narrower when the number of levels is decreased from $v=L$ to $v=1$. The parameter $K$ also influences the shrinking process of the width of the distribution of oscillation frequencies. For $K=2,3,4$, and 5, the width of the distribution seems to decrease similarly, quite rapidly, and uniformly in the consecutive coarse-graining steps in such a way that in the third step of the coarse-grained process $(v=L-3)$, the distribution of frequencies has shrunk to almost a single value. However, in the case $K=6$ (hexagons), a slower shrinking in the frequency distribution width can be seen for the first three steps, $v=L, L-1$, and $L-2$ levels, followed by a more rapid decrease from $v=L-3$ to an almost single-valued frequency. In order to measure the phase synchronization of the outputs at different steps of the coarse-graining process, for each hierarchical network, we calculate the index of synchronization occurring between $K$ outputs, which would constitute a clique in this network. ${ }^{36}$ For this, we calculate the instantaneous phase $\theta_{j}(v, t)$ of the output $s_{j}$ in the level $v$ at time $t$ as

$$
\theta_{j}(v, t)=\tan ^{-1} \frac{\dot{s}_{j}^{(v)}(t)}{s_{j}^{(v)}(t)},
$$

with $\dot{s}_{j}^{(v)}(t)$ being the time derivative of the output $s_{j}^{(v)}(t)$. Defining the phase difference $\Delta \theta_{j k}$ between two connected outputs $s_{j}^{(v)}(t)$, $s_{k}{ }^{(v)}(t)$ as $\Delta \theta_{j k}(v, t)=\theta_{j}(v, t)-\theta_{k}(v, t)$, we calculate the index of synchronization $R(v)$ between all the outputs in a clique at the level $v$ as

$$
R(v)=\left|\frac{1}{t_{f}-t_{0}} \int_{t_{0}}^{t_{f}} \frac{2}{K(K-1)} \sum_{j=1}^{K-1} \sum_{k=j+1}^{K} e^{i \Delta \theta_{j k}(v, t)} d t\right|,
$$

where the double summation is taken over all pairs of outputs in the clique, $t_{0}$ and $t_{f}$ are, respectively, the initial and final time of observation, and $i=\sqrt{-1}$. 

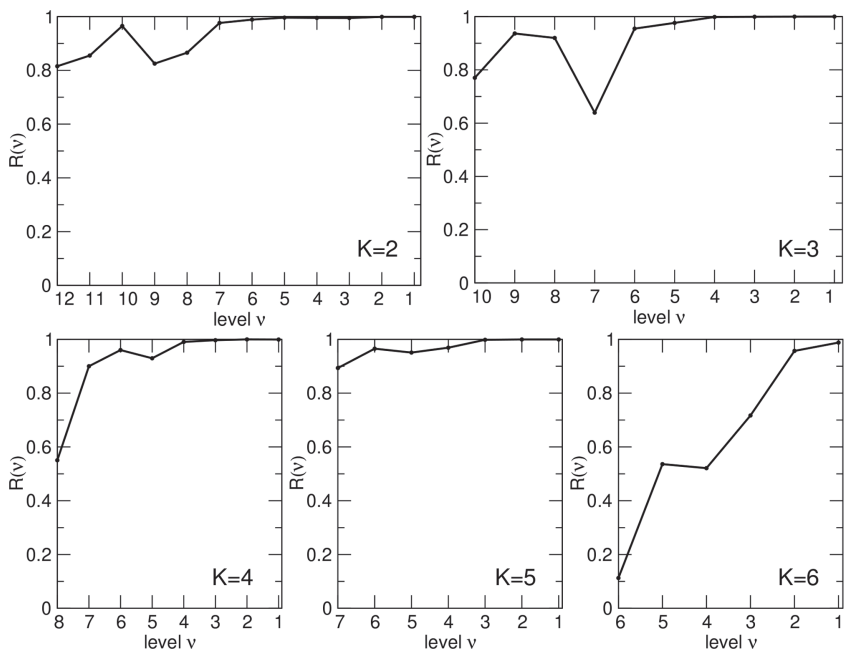

FIG. 6. The index of synchronization $R(v)$ as a function of the level $v$ for hierarchical networks with different values of $K$. The total time was $t_{f}=100$ periods, with an initial transient time $t_{0}=25$ periods.

In Fig. 6, we plot the index of synchronization $R(v)$ as a function of the level of hierarchy or coarse-graining, $v$, for the five different topologies with $K=2,3,4,5$, and 6 . The results indicate that for all of these topologies, the oscillators inside each clique progressively reach a synchronized state when the level $(v)$ of the coarse-graining process is high enough, wherein the units are in phase.

In the results shown previously, the choice of the parameters, in particular, the base interaction strength, $a$, and the decaying factor, $q$, was so that the system reached an asymptotic mean frequency $\bar{\omega}$ that is half of the fundamental frequency of a single van der Pol oscillator to mimic the possible relation between the circadian and semi-circadian human cycle. Nevertheless, by a proper tuning of the parameters $a$ and $q$, the mean frequency $\bar{\omega}$ of the system can take any possible value in the interval $\left[0, \omega_{0}\right]$, changing continuously as these parameters are varied. The extent and nature of the change in the frequency at different levels of coarsening is explored next by varying the parameters $a$ and $q$ and measuring the asymptotic mean frequency $\bar{\omega}$ that the system reaches in the final coarse-grained network. The results for five hierarchical networks (with $K=2,3,4$, 5 , and 6 and the number of levels $9,9,7,7$, and 6 , respectively) are shown in Fig. 7. For all the cases, the base interaction strength $a$ was varied from 0.1 to 0.4 , that is, from a loosely coupled to a tightly coupled system, while $q$, representing the amount of interaction decay between two consecutive levels of the hierarchy, was varied from 0.3 to 1.0 .

The results in Fig. 7 show a smooth transition between different frequencies as the parameters are changed. Inside the intervals explored, the asymptotic frequency can be tuned to any value between $0.1 \omega_{0}$ and $\omega_{0}$, and the transition is neither abrupt nor discontinuous for any value of the parameters. It can be noticed that the size of the clique of the network influences the rate at which the asymptotic frequency falls as the parameters $a$ and $q$ are varied. In
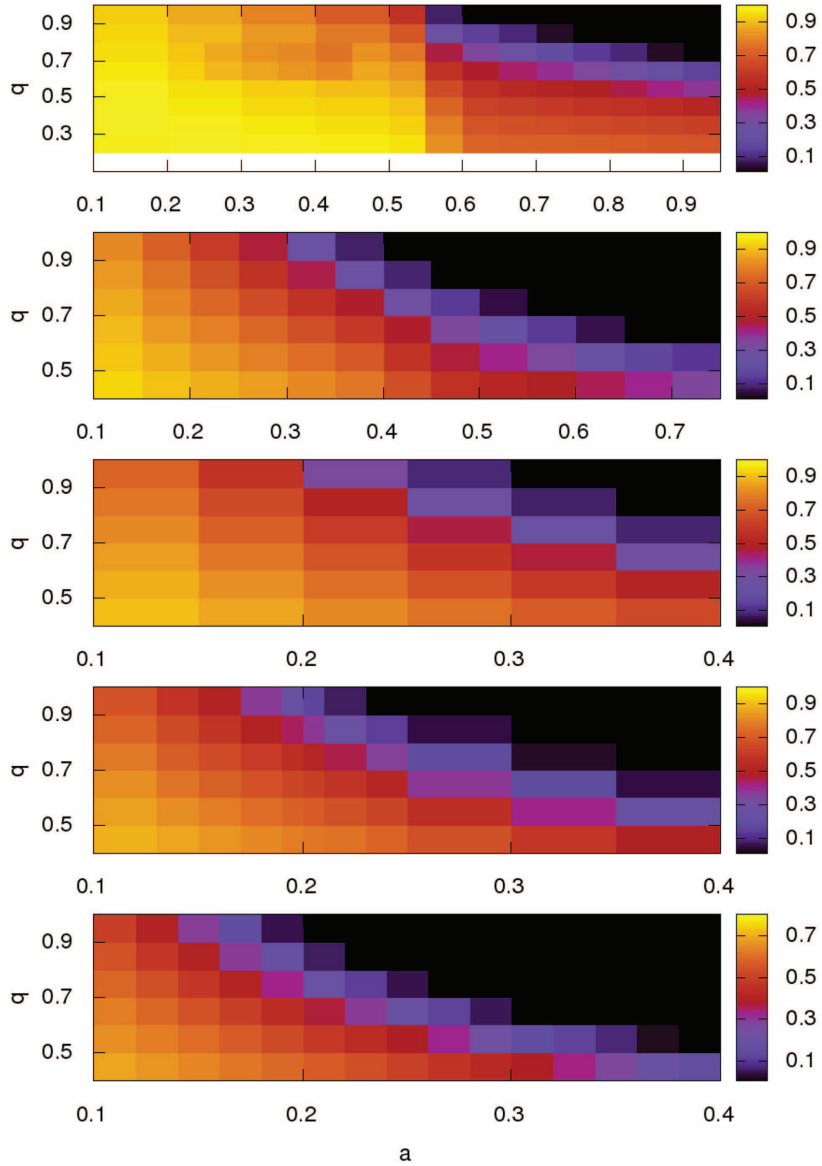

FIG. 7. Asymptotic frequency, $\bar{\omega}$, over the parameter space $(a, q)$. The frequency is in units of $\omega_{0}$. (Top) 2-cliques, with 12 levels and 512 nodes. (Middle-top) 3-cliques, with 9 levels and 19683 nodes. (Middle) 4-cliques, with 7 levels and 16384 nodes. (Middle-bottom) 5-cliques, with 7 levels and 78125 nodes. (Bottom) 6 -cliques, with 6 levels and 46656 nodes.

the case of the line and triangles $(K=2,3)$, the mean frequency $\bar{\omega}$ diminishes slowly, and larger values of $a$ and $q$ are required to force $\bar{\omega}$ to be zero, i.e., $a \approx 0.9, q \approx 0.8$ for $K=2$ and $a \approx 0.7, q \approx 0.7$ for $K=3$, respectively. On the other hand, for network topologies with larger cliques, i.e., $K \geq 4$, the decay of $\bar{\omega}$ is faster, with $K=6$ being the extreme case, where $\bar{\omega}$ tends to zero for values of $a$ and $q$ around 0.3 and 0.6 , respectively.

\section{APPROXIMATE ANALYTIC SOLUTION}

In order to gain insight into the nature of the dynamics, we construct an analytically tractable system, as an approximation. Let us consider a single oscillator and couple it with $K$ oscillators with constant strength $r_{i}(0)=a$ [level $L=0$; see $\mathrm{S}(0, \mathrm{~K})$ in Fig. 3] to obtain Eqs. (2a) and (2b). Then, adding up the coordinates of the coupled 
oscillators, we define a new set of coordinates,

$$
X_{1}=\sum_{i=1}^{K} x_{i}, \quad Y_{1}=\sum_{i=1}^{K} y_{i},
$$

which, at the level $L=1$, satisfy the following equations:

$$
\dot{X}_{1}=\omega_{0} Y_{1},
$$

$$
\dot{Y}_{1}=-\omega_{0}\left(X_{1}-r_{1}(K-1) X_{1}\right)+\mu \sum_{i=1}^{K}\left(1-x_{i}^{2}\right) y_{i},
$$

where $r_{1}=q r_{0}=q a$. In Eq. ( $\left.9 \mathrm{~b}\right)$, the second term on the right-hand side describes the non-linear part. Neglecting this, we obtain a linear system, the frequency of which is related to the frequency of the simple harmonic oscillator, $\omega_{1}^{2}=\omega_{0}^{2}\left[1-q r_{0}(K-1)\right]$. By repeating this procedure, one can construct a set of $K^{L}$ simple oscillators whose frequency is

$$
\omega_{L}^{2}=\omega_{0}^{2} \prod_{i=0}^{L}\left[1-q^{i} a(K-1)\right],
$$

which in the limit of $L \rightarrow \infty$ has a finite non-zero value. ${ }^{37}$ One recognizes the product on the right-hand side of Eq. (10) as the $q$-Pochhammer symbol $(a(K-1) ; q)_{L+1}$. The Euler function is the special case $(q ; q)_{\infty}$.

In Fig. 8, we show the frequency as a function of the level of renormalization for the cases $K=2, \ldots, 6$. Here, one finds that the frequencies obtained from the numerical solution are systematically higher than those obtained from the approximate analytical solutions, as depicted in the inset of Fig. 8. This is due to the fact that the

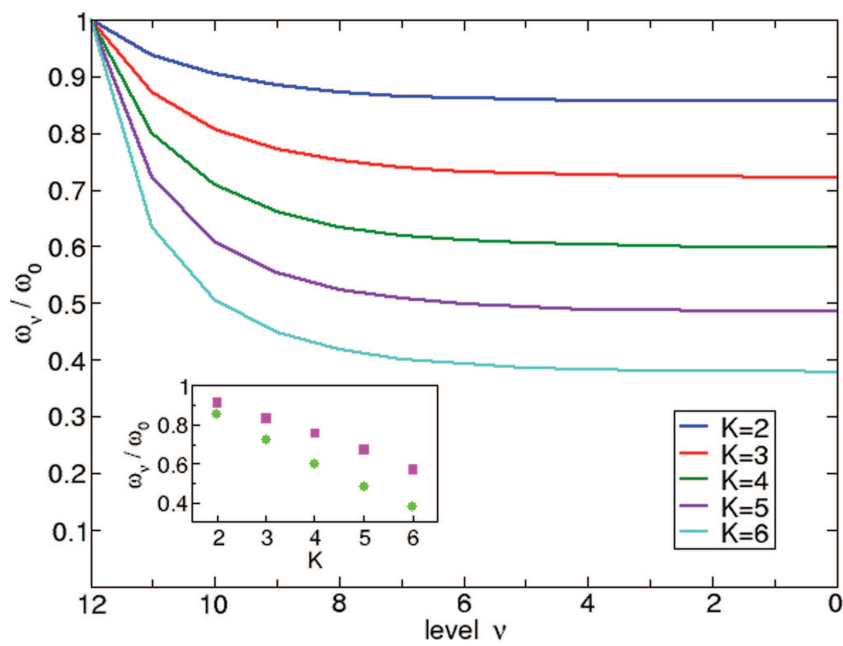

FIG. 8. Frequency $\omega$ as a function of the number of levels $v$ of the coarse-graining process, derived from Eq. (10) for $K=2, \ldots, 6$ and using the parameters $(a=0.2, q=0.70)$. The frequency is in units of the fundamental frequency $\omega(0)$ of a single van der Pol oscillator. The inset shows the comparison with the full model. Green circles refer to the analytic approximation of the frequency, and magenta squares are the numerically computed frequencies of the full model. non-linearities present in the dynamical calculations contribute by increasing the frequency from its lower limit, obtained for the linear system. We also notice that the differences between the predictions and the actual frequencies increase with $K$. This is to be expected since the number of oscillators at the base network increases enormously when $K$ is large, thus leading to the omission of correlations in the system. Nevertheless, this approximation correctly captures the qualitative dependence of the frequency on the parameters $a, q$, and $k$.

In Fig. 5, we observed that the number of frequencies different from the asymptotic mean frequency decreases rapidly as we apply more steps of coarse-graining. Thus, the assumption in our approximate analytic solution that all the units at a given level oscillate with the same frequency is well supported when the number of coarse-graining steps is large. However, to test this numerically for larger networks, such as $K=6$ and $L=12$ having more than $2 \times 10^{9}$ oscillators, would take a prohibitively long time to compute. Nevertheless, we can conclude that our approximate analytical solution describes qualitatively the main effects found in the hierarchical networks.

\section{CONCLUDING REMARKS}

Here, we have studied the dynamical properties of networks of van der Pol oscillators in fractal topologies where the strengths of the couplings decrease with successive hierarchy levels in the fractal. We have characterized the collective behavior at every level by coarsegraining the signals as outputs. It is worth mentioning that we have not only corroborated the mean-field results obtained in Ref. 17, but we have also found new properties of regular networks with hierarchical interactions, namely, synchronization and phase locking processes that emerge due to the complexity of the interactions. To our knowledge, there has been no previous study of non-linear oscillations in networks with hierarchically weighted interactions.

From the outputs at each level of the hierarchy, we have measured the mean frequency and the index of synchronization. Using these two quantities, we have demonstrated that the network can be tuned to a state of synchronization at different levels of hierarchy, with a characteristic mean frequency that is smaller than the fundamental frequency of an isolated van der Pol oscillator. In addition, the value of the mean frequency in large networks can be stabilized to any desired value lower than the intrinsic single oscillator frequency depending on the coordination number K of the network, the coupling strength, and its decay factor. These observations are supported by our approximative analytical approach, which seems to capture qualitatively the dependence of the mean frequency on the parameters.

Networks where the coupling strengths show a broad distribution are common in social and biological systems. ${ }^{12,13,38}$ Our study shows that in such networks, a robust oscillation with a precise lower frequency can be obtained if the heterogeneity in coupling strengths is introduced using a hierarchy. Note that previous studies on hierarchical coupling of oscillators were mostly concerned with topological hierarchies. ${ }^{24,39}$ Moreover, the phase locking phenomenon observed in our model is important because it allows oscillations to be reset to any desired phase while the system is evolving dynamically. It should be noted, however, that the phenomenon 
of synchronization in this system is different from the one observed in a system of weakly coupled Kuramoto oscillators. ${ }^{2,4}$

As the present study is for an idealized system, it should be followed with more realistic models including, for example, disorder in the hierarchical network structure or varying the frequency and phase of individual oscillators. In addition, it would be interesting to explore in-depth the effects of the local topology in these hierarchical systems. This could be done, for example, by introducing local defects to break the clique symmetry or by using some other fractal-like topology to test the robustness of the dynamics in less idealized situations. These could be considered realistic approaches for modeling a wide variety of networks that can be both weighted and hierarchical, such as social systems, ${ }^{40,41}$ brain networks, ${ }^{32,42}$ and networked swarms, ${ }^{43-45}$ as well as for the investigation of patterns such as chimeras. ${ }^{31,33}$

\section{ACKNOWLEDGMENTS}

D.M.-V., K.B., and K.K.K. acknowledge support from the EU's H2020 Program under the scheme "INFRAIA-1-2014-2015: Research Infrastructures," Grant Agreement No. 654024 "SoBigData: Social Mining and Big Data Ecosystem" (http://www.sobig data.eu). R.A.B. acknowledges financial support from Conacyt (Mexico) through Project No. 283279. K.K.K. also acknowledges the Rutherford Foundation Visiting Fellowship at The Alan Turing Institute, UK, Register No. 095124457 and Charity No. 1162533.

\section{DATA AVAILABILITY}

Raw data were generated at the Aalto's Triton computing server facility. Derived data supporting the findings of this study are available from the corresponding author upon reasonable request.

\section{REFERENCES}

${ }^{1}$ G. V. Osipov, J. Kurths, and C. Zhou, Synchronization in Oscillatory Networks (Springer Science \& Business Media, 2007).

${ }^{2}$ S. Boccaletti, J. Kurths, G. Osipov, D. Valladares, and C. Zhou, “The synchronization of chaotic systems," Phys. Rep. 366, 1-101 (2002).

${ }^{3}$ D. M. Abrams and S. H. Strogatz, "Chimera states for coupled oscillators," Phys. Rev. Lett. 93, 174102 (2004).

${ }^{4}$ S. H. Strogatz, "From Kuramoto to Crawford: Exploring the onset of synchronization in populations of coupled oscillators," Physica D 143, 1-20 (2000).

${ }^{5}$ A. Arenas, A. Díaz-Guilera, J. Kurths, Y. Moreno, and C. Zhou, "Synchronization in complex networks," Phys. Rep. 469, 93-153 (2008).

${ }^{6}$ P. G. Lind, J. A. Gallas, and H. J. Herrmann, "Coherence in scale-free networks of chaotic maps," Phys. Rev. E 70, 056207 (2004).

${ }^{7} \mathrm{C}$. Zhou and J. Kurths, "Hierarchical synchronization in complex networks with heterogeneous degrees," Chaos 16, 015104 (2006).

${ }^{8}$ T. Pavlidis, "Population of interacting oscillators and circadian rhythms," J. Theor. Biol. 22, 418-436 (1969).

${ }^{9}$ A. Goldbeter, Biochemical Oscillations and Cellular Rhythms: The Molecular Bases of Periodic and Chaotic Behaviour (Cambridge University Press, 1997).

${ }^{10}$ L. Glass, "Synchronization and rhythmic processes in physiology," Nature 410, 277 (2001).

${ }^{11}$ L. D. Grandin, L. B. Alloy, and L. Y. Abramson, "The social zeitgeber theory, circadian rhythms, and mood disorders: Review and evaluation," Clin. Psychol. Rev. 26, 679-694 (2006).

${ }^{12}$ D. Monsivais, K. Bhattacharya, A. Ghosh, R. I. Dunbar, and K. Kaski, "Seasonal and geographical impact on human resting periods," Sci. Rep. 7, 10717 (2017).
${ }^{13}$ D. Monsivais, A. Ghosh, K. Bhattacharya, R. I. Dunbar, and K. Kaski, "Tracking urban human activity from mobile phone calling patterns," PLoS Comput. Biol. 13, e1005824 (2017).

${ }^{14}$ R. E. Mistlberger and D. J. Skene, "Social influences on mammalian circadian rhythms: Animal and human studies," Biol. Rev. 79, 533-556 (2004).

${ }^{15}$ G. Bloch, E. D. Herzog, J. D. Levine, and W. J. Schwartz, "Socially synchronized circadian oscillators,” Proc. R. Soc. B: Biol. Sci. 280, 20130035 (2013).

${ }^{16}$ T. Fuchikawa, A. Eban-Rothschild, M. Nagari, Y. Shemesh, and G. Bloch, "Potent social synchronization can override photic entrainment of circadian rhythms," Nat. Commun. 7, 11662 (2016).

${ }^{17}$ R. A. Barrio, L. Zhang, and P. K. Maini, "Hierarchically coupled ultradian oscillators generating robust circadian rhythms," Bull. Math. Biol. 59, 517-532 (1997).

${ }^{18}$ M. Denker, M. Timme, M. Diesmann, F. Wolf, and T. Geisel, "Breaking synchrony by heterogeneity in complex networks," Phys. Rev. Lett. 92, 074103 (2004).

${ }^{19}$ A. E. Motter, C. Zhou, and J. Kurths, "Enhancing complex-network synchronization,” Europhys. Lett. 69, 334 (2005).

${ }^{20}$ A. E. Motter, C. Zhou, and J. Kurths, "Network synchronization, diffusion, and the paradox of heterogeneity," Phys. Rev. E 71, 016116 (2005).

${ }^{21}$ G. H. Paissan and D. H. Zanette, "Synchronization and clustering of phase oscillators with heterogeneous coupling," Europhys. Lett. 77, 20001 (2007).

${ }^{22}$ D. Wilson, S. Faramarzi, J. Moehlis, and T. M. R. K. Showalter, "Synchronization of heterogeneous oscillator populations in response to weak and strong coupling," Chaos 28, 123114 (2018).

${ }^{23}$ I. Pastor, V. M. Pérez-García, F. Encinas, and J. Guerra, "Ordered and chaotic behavior of two coupled van der Pol oscillators,” Phys. Rev. E 48, 171 (1993).

${ }^{24}$ S. Ulonska, I. Omelchenko, A. Zakharova, and E. Schöll, "Chimera states in networks of van der Pol oscillators with hierarchical connectivities," Chaos 26, 094825 (2016).

${ }^{25}$ P. Perlikowski, A. Stefanski, and T. Kapitaniak, "Discontinuous synchrony in an array of van der Pol oscillators," Int. J. Non Linear Mech. 45, 895-901 (2010).

${ }^{26}$ A. T. Winfree, The Timing of Biological Clocks (Macmillan, 1987), Vol. 19.

${ }^{27}$ Y. Kuramoto, Chemical Oscillations, Waves, and Turbulence (Courier Corporation, 2003).

${ }^{28}$ Z. Néda, E. Ravasz, Y. Brechet, T. Vicsek, and A.-L. Barabási, “The sound of many hands clapping," Nature 403, 849-850 (2000).

${ }^{29}$ M. J. Richardson, K. L. Marsh, R. W. Isenhower, J. R. Goodman, and R. C. Schmidt, "Rocking together: Dynamics of intentional and unintentional interpersonal coordination,” Hum. Mov. Sci. 26, 867-891 (2007).

${ }^{30} \mathrm{~S}$. Klavžar and U. Milutinović, "Graphs $s(n, k)$ and a variant of the Tower of Hanoi problem,” Czech. Math. J. 47, 95-104 (1997).

${ }^{31}$ J. Hizanidis, E. Panagakou, I. Omelchenko, E. Schöll, P. Hövel, and A. Provata, "Chimera states in population dynamics: Networks with fragmented and hierarchical connectivities," Phys. Rev. E 92, 012915 (2015).

${ }^{32}$ S. Krishnagopal, J. Lehnert, W. Poel, A. Zakharova, and E. Schöll, "Synchronization patterns: From network motifs to hierarchical networks," Philos. Trans. R. Soc. A: Math. Phys. Eng. Sci. 375, 20160216 (2017).

${ }^{33}$ J. Sawicki, I. Omelchenko, A. Zakharova, and E. Schöll, "Delay-induced chimeras in neural networks with fractal topology," Eur. Phys. J. B 92, 54 (2019).

${ }^{34}$ B. van der Pol, "VII. Forced oscillations in a circuit with non-linear resistance. (Reception with reactive triode)," Lond. Edinb. Dubl. Philos. Mag. J. Sci. 3, 65-80 (1927).

${ }^{35}$ The van der Pol equation is a special case of the Rayleigh differential equation.

${ }^{36}$ R. Biswas, K. Khamaru, and K. K. Majumdar, "A peak synchronization measure for multiple signals," IEEE Trans. Signal Process. 62, 4390-4398 (2014).

${ }^{37}$ D. Zwillinger, Table of Integrals, Series, and Products (Elsevier, 2014).

${ }^{38}$ C. Liu, D. R. Weaver, S. H. Strogatz, and S. M. Reppert, "Cellular construction of a circadian clock: Period determination in the suprachiasmatic nuclei," Cell $\mathbf{9 1}$ 855-860 (1997).

${ }^{39}$ A. Arenas, A. Díaz-Guilera, and C. J. Pérez-Vicente, "Synchronization reveals topological scales in complex networks," Phys. Rev. Lett. 96, 114102 (2006).

${ }^{40} \mathrm{~J}$. A. Davis, "Clustering and hierarchy in interpersonal relations: Testing two graph theoretical models on 742 sociomatrices," Am. Sociol. Rev. 35, 843-851 (1970). 
${ }^{41}$ A. Diaz-Guilera, "Dynamics towards synchronization in hierarchical networks," J. Phys. A: Math. Theor. 41, 224007 (2008).

${ }^{42}$ D. Meunier, R. Lambiotte, and E. T. Bullmore, "Modular and hierarchically modular organization of brain networks," Front. Neurosci. 4, 200 (2010).

${ }^{43}$ B. Bhattacherjee, K. Bhattacharya, and S. S. Manna, "Cyclic and coherent states in flocks with topological distance," Front. Phys. 1, 35 (2014).
${ }^{44} \mathrm{~A}$. Zafeiris and T. Vicsek, "Advantages of hierarchical organization: From pigeon flocks to optimal network structures," in Research in the Decision Sciences for Global Business: Best Papers from the 2013 Annual Conference (Pearson Financial Times Press, Indianapolis, IN, 2015), pp. 281-282.

${ }^{45}$ K. P. O'Keeffe, H. Hong, and S. H. Strogatz, "Oscillators that sync and swarm," Nat. Commun. 8, 1-13 (2017). 